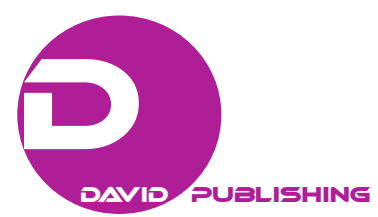

\title{
Emerging Technologies As Drivers for Developing Organizational Innovation Competencies
}

\author{
Maria José Sousa \\ Universidade Europeia, Lisboa, Portugal
}

\begin{abstract}
This article will analyze importance of emergent technologies as drivers for developing organizational innovation competencies. The relevance of this research is based on the approach to emergent technologies articulating them with the competencies development models and organizational innovation theories. To formulate the research problem, two analytical dimensions were considered: emergent technologies and organizational innovation competencies anchored to the following research question: What are the organizational innovation competencies that rise from emergent technologies adoption by companies? Based on the literature review of prospective studies conducted in economic clusters in Portugal, the organizational innovation competencies that raised from the adoption of emerging technologies were investigated and identified: internet, mobile technologies, artificial intelligence, big data, robotics, and nanotechnologies. The methodology used was document analysis, based on the prospective sectorial studies on Portuguese industry.
\end{abstract}

Keywords: competencies, innovation, organization, prospective, emerging technologies

\section{Introduction}

Technologies are technical innovations that have the potential to change and restructure organizations and promote the emergence of new businesses.

To evaluate the impact of technologies in organizational innovation competencies first, it's necessary to analyze how they affect industry and organizations.

Internet, mobile technologies, artificial intelligence, big data, robotics, and nanotechnology to be potentially disruptive technologies and the main phenomena that are changing the industry can be considered.

Internet has changed the music, travel, and retail industries. Mobile technologies are changing services sector as e-tourism, e-health, e-marketing, and education. Artificial intelligence is changing the whole industry, mainly areas that need close collaboration between humans and computers. Big data is changing the way people store, organize, and visualize information. Robotics is transforming industry, mainly automotive, aeronautic, health care, and also engineering. Nanotechnology is changing computer industry, energy industry, and health industry.

Mainstream technologies meet customers' needs, but don't have the impact of new emerging technologies. They can be currently regarded as part of a strategy to boost the economy and the development of

Maria José Sousa, Ph.D., Universidade Europeia, Lisboa, Portugal.

Correspondence concerning this article should be addressed to Maria José Sousa, Universidade Europeia, Estrada da Correia, 53, 1500-210 Lisboa, Portugal. E-mail: maria-jose.sousa@europeia.pt. 
organizational innovation competencies which are crucial to achieve that goal.

The main objective of this research was to identify organizational innovation competencies that are arising from the emergent technologies adoption by companies. The identification and development of that kind of competencies are a challenging task, either internally within organizations or externally, by the creation of public policies on a complex economic environment.

In the context of this article, the focus will be in the identification of sets of competencies by the organizational functional areas.

The article briefly explores the concepts of competencies, emergent technologies, and innovation, followed by the presentation of the methodology used for the competencies identification. The article concludes with the research findings.

\section{Literature Review}

\section{Emergent Technologies}

As emerging technologies, internet and cloud computing, mobile technologies, artificial intelligence, big data, robotics, and nanotechnologies will be considered.

Internet and cloud computing is a model for delivering internet based information and technology services in real time or "on-demand" and is particularly driven by: economic opportunity; cost reduction; technological change; and consumer's needs. The current economic climate is seen to be a strong driver with cloud computing enabling companies to not only move into new markets, reduce costs, and become more agile but also to provide growth through the enablement of new business models.

A wide range of technical skills are needed for cloud computing, although security, networking, virtualization skills, and big data analytics are considered to be the key skills for cloud. However, IT specialists need broader business skill-sets, especially risk management and business stakeholder management, to bridge the divide between IT and wider business operations.

Mobile technologies englobal communications, devices, and applications that are integrating, converging, and enabling multiple uses and advanced collaboration. This requires constant innovation and new technology solutions. There are significant challenges for IT specialists managing mobility, but mobile technologies bring new operational models to businesses, providing opportunities for growth and more efficient working methods for all employees. Mobility is increasingly a thriving part of digital sector products and services with massive potential for growth, and an area where significant recruitment and retention pressures are emerging, particularly in the recruitment of mobility specialists who need extensive technology experience together with specific mobile technology skills.

Artificial intelligence is the development of computer systems able to perform tasks that normally require human intelligence and is being applied to almost all situations in high tech industry. It requires specialized technological skills, but most of all behavior skills like ethics and news capacities to interact with new forms of live.

Big data as new phenomenon is associated to the increase of volume of data, as a result of internet, social media, and mobile devices. The potential value of this data has led to the development of new techniques for managing and analysing very large data sets, such as images, text, or speech. Competencies like data analysis, data selection, and data security assume a big relevance to all employees of an organization.

Robotics by integrating technologies, such as computer vision and automated planning with very small, 
high-performance sensors, actuators, and cleverly designed hardware, have given rise to a new generation of robots that can work alongside people and flexibly perform many different tasks in unpredictable environments. Examples include unmanned aerial vehicles that share jobs with humans on the factory floor, robotic vacuum cleaners, and a slew of consumer products, from toys to home helpers, requiring operational technological competencies and also programing skills.

Nanotechnology is engineering of functional systems at the molecular scale and may be implied by the term "automation", including:

- faster actions and decisions (for example, automated fraud detection, planning, and scheduling);

- better outcomes (for example, medical diagnosis, oil exploration, and demand forecasting);

- greater efficiency (that is, better use of high-skilled people or expensive equipment);

- lower costs (for example, reducing labor costs with automated telephone customer service);

- greater scale (that is, performing large scale tasks impractical to perform manually);

- product and service innovation (from adding new features to creating entirely new products).

These technologies are changing the way markets and organizations work. An emerging theme is the growth of "bring your own device" (BYOD) where employees are driving change in the workplace

\section{Innovation in Organizations}

The main driver for innovation is technology and organizations are facing enormous changes, because the high level of technology is adopted by industries. This context is important to analyse the concept of innovation.

Innovation can be translated as "the successful production, assimilation, and exploitation of novelty", according to the Green Paper on Innovation from the European Commission (1996).

The concept is structured around three pillars: the renovation and enlargement of the range of products and services and the associated markets; the creation of new methods of production, supply, and distribution; and the introduction of changes in management, work organization, and skills of the workforce. The object of this research fits the last vector-organizational innovation.

According to Kovács (1989), organizational innovation means applying new principles to production of goods and services, new structures and processes, new kind of relationship between people and role models (values, attitudes, and mind-sets). The innovation is supported on tacit knowledge (rooted in people experiences and insights) whose costs and benefits are harder to quantify and observe then explicit knowledge (enclosed in documents, reports, memos, and data bases).

Systems theory and other approaches, such as complexity theory (Stacey, 2001), have contributed to open new dimensions for organizational innovation.

The concept of the innovation itself can be viewed from different perspectives, making a existence of a single definition difficult, however, there is a consensus on the fact that innovation refers to something new.

Another main idea is that innovation should be something useful (Gronhaug \& Kaufmann, 1988; Padmore, Schuetze, \& Gibson, 1998; Cooper, 1998), this assumption differs innovations of inventions that may not have practical application.

With regard to organizational innovation (Gjerding, 1996), it states that the main objectives of its implementation in companies are increasing effectiveness and efficiency of work, cooperation and coordination within the company, the company's ability to adapt to change, the ability to develop new products and services, and the ability to generate, transferr, and appropriate knowledge and develop their organizational skills. 
According to Tidd, Bessant, and Pavitt (2005), there are some factors which can be more favourable and open to the innovations - training and development of employees, organization of work, involvement of people in the innovation process, and how the company learns and shares knowledge.

The knowledge plays a major role in the organizations development and has been studied by several authors. Polanyi (1998) stated that knowledge is the ability to act and explained that knowledge is created by the flow of information associated with the beliefs and commitments of its holder.

In most companies, the process of knowledge creation is accidental and unpredictable. While there may be cases of companies that have implemented management systems knowledge, which facilitates the generation, transfer, and appropriation. In general, within the universe of the company, the organizational knowledge creation takes place in the whole team, by analysing individual potential and combinations of the team members.

The individual knowledge starts to set up as organizational knowledge when is shared in the organization.

In view of Nonaka and Takeuchi (1997), knowledge is created within the company to make it more successful, keep it in the market, and be ahead of their competitors.

Through the generation and sharing of knowledge, competencies that allow the company to compete in the market can be developed.

\section{Concept of Competencies}

In the $80 \mathrm{~s}$, the concept of competencies starts to have a big importance, due to technological, organizational, and economic factors. It begins to be considered as a resource of individual and organizational nature which would allow competitiveness and productivity advantages to companies.

The complexity and the uncertainty, partly due to the globalisation and accelerated rhythm of technological change, demand human resources with competencies that help the organizations to overcome the appearing challenges.

In this context, Gilbert and Parlier (1992) defined competencies as a set of knowledge, capacities of action and behaviours, and structured according to an objective in a specific situation.

Meanwhile, as a result of the changes occurred in organizational contexts, other concepts emerged associated with the concept of competencies. In this way, the concept is created of organizational competencies which are considered by Prochno (2001), as a co-ordination of different basis of knowledge (know-how, know-what, and know-why) and its application to one (or more) product(s) or process(es).

In the same way, the concept of individual competencies has been studied by different authors, for example, Lopes (1999), who built a typology of competencies based on the typology of Boterf (1999). This typology distinguishes the following types of knowledge and capacities in competencies:

- theoretical knowledge: which integrate the concepts, the subject knowledge, the organizational and rational knowledge, and also the technical knowledge about the context, processes, and operational methods and means;

- know-how: which is related to the ability to execute operations, use instruments, and apply methods and procedures. These know-how have an operational character, of practical application or operationalization of theoretical knowledge;

- social and relational know-how: which is related to attitudes and personal qualities and to the predisposition to act and interact with others, i.e., the ability to co-operate with others; 
- cognitive capacities: which are related to intellectual operations, that can be simpler (enumerate, compare, define, and describe) or more complex (inductive generalisation, constructive generalisation, analogical reasoning, and abstract reasoning). They describe capacities of combining different knowledge, of coordinating actions, so that solutions are found and problems are solved.

Historically, the word competencies have been used to refer individual characteristics. However, in the concept of Boterf (2001), although the competencies always refer to the individual, all of them have two dimensions: the individual and the collective (organizational).

In this way, the concept of competencies assumes a rather large scope which makes it complex and makes its comprehension/understanding and concept delimitation difficult.

Green (1999) tried to contribute to the clarification of the concept of competencies by presenting some different senses it can assume (Figure 1).

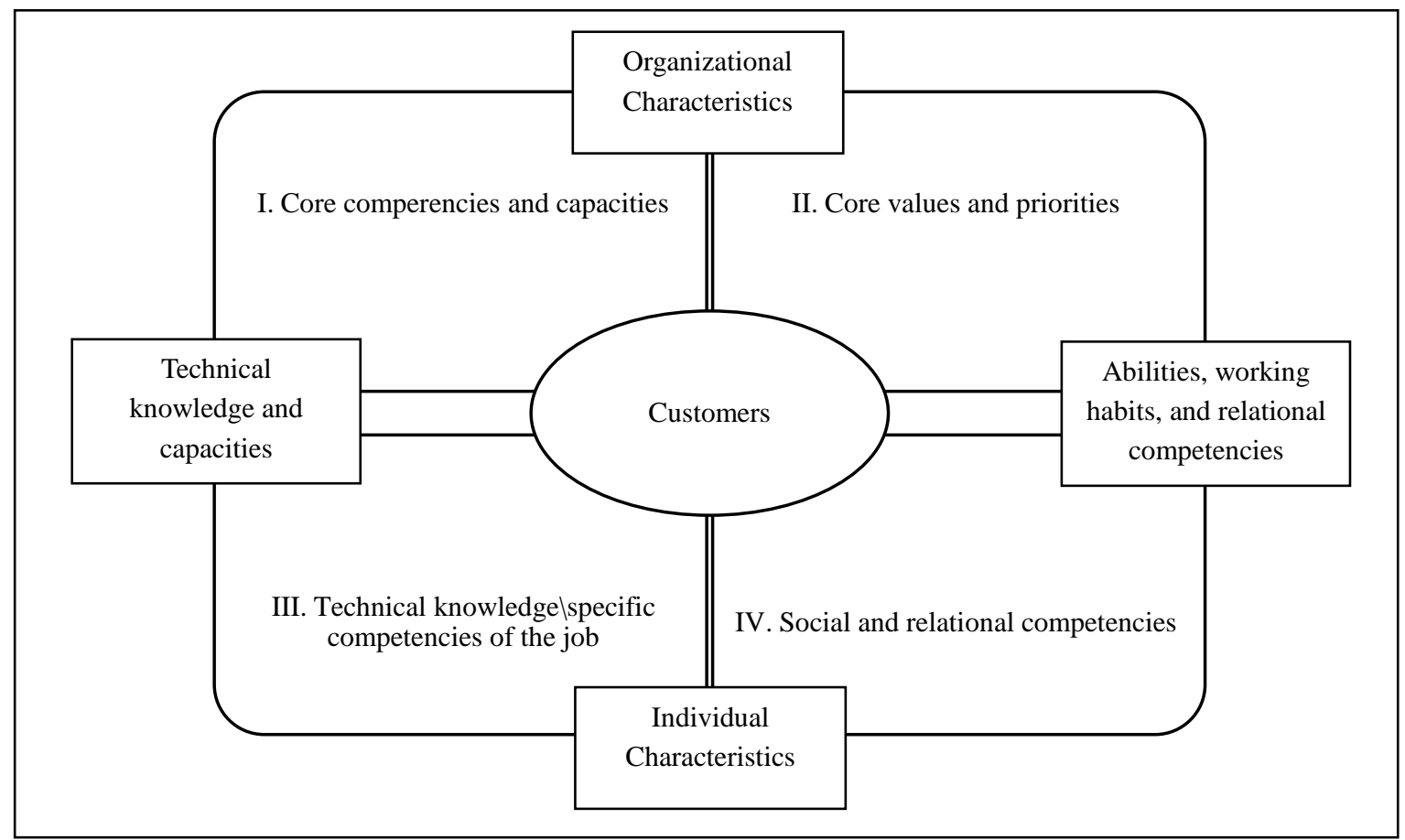

Figure 1. Competencies scope.

Organizational characteristics. The organizational characteristics reflect the identity of the organization in which the mission, the values, and its own culture are inserted. This holds the core competencies and capacities that are reflected in the mission and in the vision of the organization, beyond the values and core priorities that are reflected on the shared working habits and in the handbooks of conduct and ethics.

The core competencies are the strategic competencies, which make an organization unique and distinctive. They can be, for instance, a technical knowledge or a specific technology which can offer a unique value to the customers and that distinguishes the enterprise from the rivals. They are the basis for the organization to develop beyond its final products.

A core competence is the technical know-how, which is of the outmost importance for the objectives of the organization. They are a source of competitive advantage which is the result of the value acknowledged by the customers and it is difficult to imitate. 
The core capacities are also very important for the effectiveness of the organization and are easily understood by the customers - they are a set of business processes strategically understood (Stalk, Evans, \& Shulman, 1992).

The values and priorities of the organization aim to create a sense of community, which can lead to an increase of trust and commitment by the workers.

They complement the technical aspects of the work and explain the reason why the work is accomplished. They imply the sharing of beliefs and cultures, including behavioural rules.

The priorities reflect the effort of the organization to use individual competencies, such as working habits, people's knowledge to fulfill the business and to make the working systems function, in a more efficient and effective way.

An important priority of the enterprise is its will to promote the participation of the workers, in order to develop its performance (Lawer, Mohrman, \& Ledford, 1995).

Individual characteristics. The individual characteristics change the performance of the individual, because they reflect themselves in the content of his/her work.

In what regards the individual characteristics, the technical knowledge/specific competencies of the job and the social relational competencies may be identified.

Technical knowledge/Specific competencies of the job. These types of knowledge are learnt in formal learning situations and differ, according to the specificity of the job and the sectors of activity. They are the basis of the core of strategic competencies of organizations.

Relational and social competencies. These competencies include working habits, communication styles, leadership forms, and teamwork. They are competencies easily transmissible between jobs and even sectors of activity. However, they vary from organization to organization, according to the importance that each one gives to certain competencies or to leadership/management styles adopted.

The social and relational (or behavioural) competencies are used/developed in the execution of job related tasks, but are supported to the core values and priorities of the organization.

Both the individual and the organizational characteristics can have a strategic aspect and contribute to the creation of value, generating advantages acknowledged by the customer: fastness of response, precise demands of quality, and after-sales service - therefore, it is speaking about strategic competencies.

In the same line of thinking, Green (1999) spoke of critical competencies, considering three criteria: strategy, competitiveness, and specificity or rarity:

- The criterion strategy takes into account the indispensable competencies to the strategic orientations of the organizations;

- The criterion competitiveness allows to identify the necessary competencies to acquire or keep a competitive advantage in a domain of activity;

- The criterion specificity has to do with the specific characteristics of the enterprise and that are not found in the working market.

Despite the duality that competencies assume, in last analysis, they are a set of individual and/or group knowledge that allow to generate orientation to ensure sustainability and competitiveness of the organizations.

\section{Contexts Influencing Competencies Development}

In the last years, the organizations have faced an increasing competition, budgetary constraints, and a 
major technological change. To face these changes, many have developed practices, policies, and processes that have an increased value to the customers, reflected for instance in the improvement of service quality, reduction of the costs, or a bigger fastness in the product delivery/service accomplishment.

Influenced by these changes, the organizations have been suffering structural changes, implying the need to develop new competencies. Figure 2 presents a scheme that intends to show the relationship between the emerging technologies impact on organizations and on competencies.

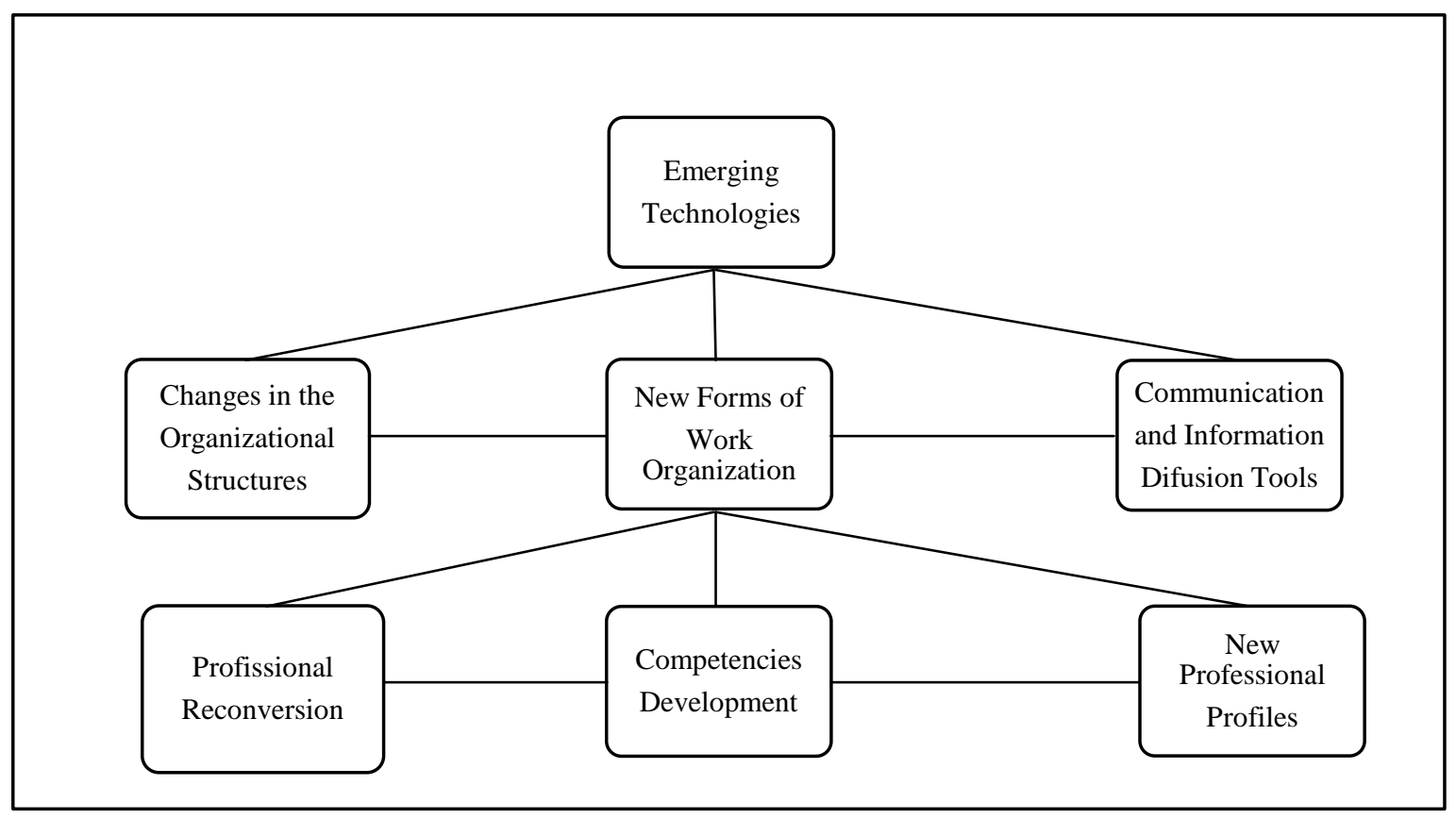

Figure 2. Emerging technologies impact on competencies development.

Different empirical studies have revealed that the technologies lead to changes in the structures of organizations. It can be observed that work organization, mainly structured by multiple functional levels, has tended to change into a horizontal model, where all the workers play a key role in what concerns the taking of decision and the organization of his/her own work.

The same studies confirm that there is an orientation for the creation of work groups/teams, instead of individual work, emerging a need of work co-ordination instead of a culture of control and centralisation of decisions. These changes imply that the elements of the teams need to possess competencies, the necessary information autonomy to respond to unpredictable disturbances, resulting from of the technical systems and of the environment.

The work begins to be organised in turn of the processes and not of the jobs, implying not only team work but also, and most importantly, the responsibility taking by each of the workers for the quality of the developed work and for the achieved goals. These developments require competencies at the level of technical knowledge and especially at the level of relational/social knowledge.

Already in 1989, Kovács approached the anthropocentric model which assumes the development of specific structures conceived, according to the valuableness of the human factor and new organizational principles, such as the autonomy, responsibility, creativity, professionalization, decentralisation, participation, and co-operation, being demanded new technical and social knowledge for each level of qualification: 
- superior management staff: They must have a systemic vision, a strategic management, new methods and techniques of management, and capacity of sharing information, listening, negotiating, and motivating;

- superior technical staff connected with new technologies: They must have knowledge of "hardware" and/or "software", as well as "orgware", co-operation with specialists connected with the social system, so that the integration of the IT in the organizations is possible;

- medium leadership: More planning, greater co-ordination, and less direct control are required. Beyond larger technological knowledge, they must have motivation for the formation, communication, and co-operation, which presumes higher technical formation in the areas of organization, management, and human relationships;

- operators: They should be polyvalent, have initiative and responsibility spirit and capacity of identifying and solving problems, know how to communicate and work in team, and have also to achieve quality control.

This organizational paradigm appeals to organic flexible structures, continuously adaptable to new situations presented, in opposition to bureaucratic and centralised structures. In this paradigm, the enterprises tend to be innovating and more receptive to changes. They implement new forms of organising work and present decentralised and participative models of decision, appealing to a greater autonomy and responsibility by their collaborators. However, to achieve an organizational development to this level, an investment must occur in the development of the competencies of people who form the organization.

\section{Methodology}

The methodological approach of the research was qualitative or intensive. With regard to the specifics of this research, the main technique used was content analysis from the literature review of prospective studies on organizational innovation competencies. This methodology was used to analyze the presence of certain words or concepts within the studies to identify competencies which can boost innovation in organizations.

This is an exploratory and descriptive study comprising the data collection, analysis, and synthesis of results arising from research in Portuguese prospective studies developed in industrial sectors using a systematic analysis.

The search was based on the keywords "competencies", "innovation", and "technology" and the period considered was between 1999 and 2014.

\section{Research Findings}

\section{Organizational Innovation Competencies}

The research found a set of competencies that raised from the emergent technologies and that conducted to several changes in organizations.

The following set of competencies were identified across industrial sectors and organized by functional areas:

(1) management: (a) competencies at the level of the definition of business strategies which add value to the offer starting from immaterial factors; (b) competencies at the level of the definition of internationalisation strategies (software to identify and analyse potential markets, to select and implement ways of entrance in the markets, and to define product types to internationalise); (c) capacity to manage strategic deals and alliances with enterprises of the sector (internet and social and mobile technologies allow the creation of global networks to give response to more demanding orders) or of other activity sectors (for instance, to present solutions); (d) capacity to diversify the business area, identifying new business opportunities, investment analysis, and 
economic viability studies through the use of analytical models created, according to the premises of artificial intelligence and big data; (e) social and relational competencies, in what concerns the capacity of communication, leadership, and interpersonal relationships, and also virtual interrelations among managers, employees, and also partners; and (f) competencies related to the management of people and their development, using information systems and the potentialities of cloud computing.

(2) commercial/marketing: (a) competencies at the level of information analysis which allows to identify market evolution tendencies (software that allows market studies, statistics, and management reports); (b) competencies at the level of analysis and syntheses of information related to products (technical characteristics, ways of using...) and of document elaboration with the discrimination of selling procedures (product presentation and argument factors among others); (c) competencies about the technical characteristics of the manufactured products, due to the necessity of bigger capacities of technical and counseling arguments, as well as the service of after-sales technical assistance, using digital marketing facilities, like CRM (customer relationship management) or BI systems (business intelligence); (d) competencies concerning knowledge of products of other activity sectors, due to the staking on integrated solutions; (e) bigger demands on competencies associated with potential customers identification and with the deep knowledge of their necessities (to know the search profile); (f) competencies regarding the use of different selling techniques (ways of approaching, commercial argumentation and presentation techniques, and response to customers objections...); and (g) social and relational competencies, due to the necessity of co-operating with other different enterprise areas, in order to transmit information, suggestions, and complaints obtained from the customers.

(3) management production: (a) competencies associated to robotics, nanotechnology, and also software that allows production management in a very effective way; (b) competencies associated with the redrawing of productive layouts, through the contribution of the various enterprise areas and of the suppliers themselves, to create more adequate work flows within the different areas of the production process, in a way that it allows the rational circulation of materials, reducing the non-productive times and consequently the costs associated to the production; (c) competencies associated with the organization of the working conditions, having in mind the rationalisation of the investments in equipment and guaranteeing the observance of the aspects related to the hygiene system and security at work; (d) competencies associated with the analysis of information related to productivity, in what concerns manpower costs, production costs, quality problems in the process and in the product, stock management (raw material, materials, or finished products) among others; (e) competencies related to the possibility of implementing new models of work organization, recurring to models like the just in time and the total quality management, taking into account line the costs of production and deviation of productivity; (f) competencies associated with: (a') the adaptation to change (raw materials, materials, technology, and products); (b') the development, continuity, and support to more flexible forms of work organization (working posts rotation and group work); (c') the organization and division of work, according to the characteristics of the work organization and of the individuals; and (d') the capacity of developing social and relational knowledge which allows the co-ordination of working teams, taking advantage of all the potential of its elements; $(\mathrm{g})$ competencies associated with the knowledge of the subcontracting market, which allow to select enterprises and subcontract them according to production capacities (quantity, quality, deadlines, and production costs); (h) competencies related to the quality rules applicable to the sector and to the specificity 
of the quality system implemented in the enterprise; and (i) competencies related to the area of security at work, in what concerns the implementation and the use of individual security mechanisms.

(4) quality: (a) competencies at the level of implementation and management of a quality management system; (b) competencies in the diagnosis of the causes for the non-conformity in the products/materials, in the different phases of the productive process, namely in the reception of raw materials; (c) competencies associated with the definition of quality control mechanisms; (d) higher demands at the level of the adaptation of rules and procedures depending on the specificity of products, productive process, and technology; (e) social and relational competencies due to the necessity of co-operation with the suppliers (for the definition of quality requirements related to material) and with the customers (for the definition of quality rules related to products to produce), and also with the other areas of the enterprise: (a') conception and development, for the determination of quality rules of new products which are being conceived; (b') technical assistance, so that through the elaboration of an information syntheses about the quality rules of products, the professionals who give the technical assistance to products can proceed and inform the customers adequately; (c') production, so that the product fabrication can be made according to the defined quality rules; and (d') commercial/marketing, for defining a commercial and marketing policy which has into account the quality of the products as a distinctive factor of competitiveness, not only at the level of product promotion campaigns, but also to the level of the techniques and selling arguments used; and (f) competencies at the level of environmental requirements, so that these may be considered as fundamental elements when defining quality rules of products.

(5) production: (a) competencies associated to robotics and Artificial intelligence, mainly through the operation of the machinery of the plants; (b) social and relational competencies, due to new forms of work organization; (c) competencies associated to the greater necessity of polyvalence of the professionals of the production area, so that they can operate different types of machines; (d) competencies associated with new forms of work organization, in what regards the methods of teamwork, flexibility to adapt to changes in the working processes (as a response to the high rhythm of innovation), knowledge about different types of technologies (of different generations and with different uses...), such as it is the case of the progressive tendency for the use of flexible working cells and of teamwork; (e) competencies regarding a bigger initiative, decision taking, and responsibility assuming; (f) competencies regarding the quality control and specially regarding the patterns of quality and technical specificity of products; $(\mathrm{g})$ competencies associated with the hygiene procedures and security at work in general, and in particular regarding the use of individual protection equipment.

\section{Future Research}

New expectations of innovation competencies are emerging, as this research field is becoming strategically important for organizations. In this context, a consistent framework needs to be developed:

- Develop other aspects in the future, directly related to the findings from this research;

- Develop a theoretical model of reference that summarizes and systematizes the concept of organizational innovation, as well as concepts related to knowledge and skills;

- Identify and analyse the processes of competence development used by companies;

- Construct a typology of forms of organizational innovation which will enable help build a framework for analysis of the practices of organizational innovation and help create models of implementation;

- Create a competencies development model associated to the emergent technologies to facilitate the 
implementation and diffusion of organizational innovation in companies in order that they become more competitive.

\section{Conclusions}

This research tries to make an approach to the innovation competencies to be developed and that rises from the adoption of emergent technologies by organizations.

This research creates a model of competencies development, identifying competencies that rises from technology adoption in the most relevant areas of an industry.

Current trends in technology allows industries to build products based on machine learning, natural language processing, computer vision, or robotics automating business processes and enhancing their products and services. Also way organizations work and interact with the market are changing and impacting the competencies of theirs workers.

Technological progress should expand the impact of technologies, a growing number of organizations will likely find compelling uses for them, and organizations may find innovative applications that will improve their performance or develop new competencies, enhancing their competitive position.

\section{References}

Boterf, G. L. (1999). L'ingénierie des Compétences. Pairs: Éditions d' Organization.

Boterf, G. L. (2001). Construire les competences individuelles et collectives. Pairs: Éditions d' Organization.

Cooper, J. R. (1998). A multidimensional approach to the adoption of innovation. Management Decision, 36(8), $493-502$.

European commission. (1996). The green book on innovation. Brussels: EU.

Gilbert, P., \& Parlier, M. (1992). La compétence: du "mot-valise" au concept opératoire. Actualité de la formation permanente, $116,14-18$

Gjerding, A. N. (1996). Organisational innovation in the Danish private business sector (Druid working paper No.96-16, Department of Business Studies, Aalborg University, Aalborg).

Green, P. C. (1999). Building robust competencies: Linking human resources systems to organizational strategies. San Francisco: Jossey-Bass Publishers.

Gronhaug, K., \& Kaufmann, G. (1988). Innovation: A cross-disciplinary perspective. Oslo: Norwegian University Press.

Kovács, I. (1989). Tendências de transformação tecnológica e organizacional nas empresas: A emergência de novos sistemas produtivos. Revista do Centro de Estudos Economia e Sociedade, 1, 39-66.

Lawer, E. E. I., Mohrman, S. A., \& Ledford, G. (1995). Creating high performance organisations: Practices and results of employee involvement and total quality management in Fortune 1000 Companies. San Francisco: Jossey-Bass.

Lopes, H. I. (1999). Estratégias Empresariais e Competências-Chave (Dinâmia, ISCTE).

Nonaka, I. E., \& Takeuchi, H. (1997). Criação de Conhecimento na Empresa. R. Janeiro: Ed. Campus.

Padmore, T., Schuetze, H., \& Gibson, H. (1998). Modeling systems of innovation: An enterprise-centered view. Research Policy, 26(6), 605-624.

Polanyi, M. (1998). Personal knowledge. Towards a post critical philosophy. London: Routledge.

Prochno, P. (2001). Relationships between innovation and organizational competences. Proceedings from Academy of Management Annual Meeting, Washington, D.C., USA.

Stacey, R. (2001). Complex responsive processes. London: Routledge.

Stalk, G., Evans, P., \& Shulman, L. E. (1992). Competing on capabilities: The new rules of corporate strategy. Harvard Business Review, 70(2), 57-69.

Tidd, J., Bessant, J., \& Pavitt, K. (2005). Managing innovation: Integrating technological, market and organizational change (3rd ed.). Chichester: John Wiley and Sons Ltd. 